\title{
Determination of Incremental Local Pulse Wave Velocity Using Arterial Diameter Waveform: Mathematical Modeling and Practical Implementation
}

\author{
Nabeel P M ${ }^{1,2}$, Raj Kiran $V^{1}$, Jayaraj Joseph ${ }^{2}$, Mohanasankar Sivaprakasam ${ }^{1,2}$ \\ ${ }^{1}$ Indian Institute of Technology Madras, Chennai, India \\ ${ }^{2}$ Healthcare Technology Innovation Centre, Chennai, India
}

\begin{abstract}
Background and Aim: Given the knowledge of the noninvasive assessment of local pulse wave velocity (PWV) for cardiovascular risk stratification, it is apparent that it is necessary to develop a practically feasible solution to measure and trace instantaneous variations in local PWV (incremental local PWV) from the target arteries.

Methods: From the arterial blood pulse propagation characteristics, wave nature of the transmural pressure, and the distending vessel wall geometry, a mathematical model was developed to evaluate incremental local PWV using arterial diameter waveform. Its practical feasibility and the measurement accuracy were demonstrated invivo using a custom image-free ultrasound device, with the Bramwell-Hill method as the reference.

Results: The proposed technique and developed device reliably captured incremental local PWV from the carotid artery. The locus of instantaneous variations in carotid local PWV obtained using the developed model traced the reference values, with a root-mean-square-error lesser than $0.05 \mathrm{~m} / \mathrm{s}$. Study results further established the practical feasibility and accuracy of this novel approach.

Conclusion: The theoretical basis and measurement method of this work is a solution for non-invasive, realtime assessment of incremental local PWV and its locus.
\end{abstract}

\section{Introduction}

Clinical importance of the blood pulse wave velocity (PWV) measured over small arterial segments, known as the local PWV, is well established in recent years $[1,2]$. Typically, local PWV from target arterial sites (artery under clinical investigation) is evaluated for a single and the most convenient fiduciary point from each cardiac cycle and averaged over the measurement period. Although such single-value estimates are associated with various pathophysiological conditions [1], instantaneous variation and the degree of change in local PWV within a cardiac cycle have recently been recognized as key measures for advanced cardiovascular screening and diagnosis [3-5]. Physiologically, the instantaneous variation in local PWV occurs due to the dependency of vessel elasticity on the arterial transmural pressure (blood pressure (BP)) [6]. More specifically, arteries become momentarily stiffer at higher distending pressures; higher the vascular stiffness and BP level, faster the blood pulse wave propagation. During the cardiac cycle, the pressure in arteries increases in the anacrotic phase, resulting in an increase in local PWV as a function of time (t). This phenomenon is referred to as 'incremental local PWV'.

Non-invasive measurement of incremental local PWV and assessment of the degree of variation over a cardiac cycle is practically challenging using the currently available methodologies [1]. However, considering the clinical importance of incremental local PWV [2], it is essential to introduce a practically feasible solution to assess the instantaneous value of local PWV and trace the locus over continuous cardiac cycles. To our knowledge, the work presented in this paper is the first attempt that has been made to introduce an empirical model to deduce the locus of incremental local PWV over the cardiac cycle using directly measurable physiological parameters. A detailed discussion on the modeling and the method for practical implementation is presented in the following sections, followed by in-vivo validation.

\section{Theoretical analysis}

\subsection{Modeling of incremental local PWV}

Since arteries are essentially hyperelastic, the uniaxial stress-strain relation changes with the tension experienced by vessel walls due to the transmural pressure. Therefore, the stress-to-strain ratio is non-linearly dependent on the instantaneous arterial pressure $\left(\mathrm{P}_{(\mathrm{t})}\right)$, and consequently the arterial geometry. The nature of $\mathrm{P}_{(\mathrm{t})}$ and circumferential geometrical variations has long been recognized. In 1980, Hayashi et al. have proposed a heuristic model illustrating the functional relationship between distending arterial pressure $\mathrm{P}_{(\mathrm{t})}$ and the lumen diameter $\mathrm{D}_{(\mathrm{t})}$ [7]. This nonlinear relationship of $\mathrm{P}_{(\mathrm{t})}$ and $\mathrm{D}_{(\mathrm{t})}$, as presented in (1), is directly applicable for individual cardiac cycles for a wide physiological pressure ranges. The exponential term $\beta$ is 
indicative of vessel wall stiffness and usually referred to as 'arterial stiffness index'. As per the definition, the value of $\beta$ within a cardiac cycle, corresponding to a reference pressure $P_{R}$ and luminal diameter $D_{R}$, does not depend upon the $\mathrm{P}_{(\mathrm{t})}$ at the time of measurement $[7,8]$.

$$
P_{(t)}=P_{R} e^{\beta\left(\frac{D}{(t)}-1\right)}
$$

While performing measurements on individual cardiac cycles, their diastolic pressure $\left(\mathrm{P}_{\mathrm{D}}\right)$ and corresponding end-diastolic diameter $\left(\mathrm{D}_{\mathrm{D}}\right)$ values can be used as reliable estimates for $P_{R}$ and $D_{R}$ respectively. Therefore, (1) can be further expressed, for a given cardiac cycle, as in (2).

$$
P_{(t)}=P_{D} e^{\beta\left(\frac{D_{(t)}}{D_{D}}-1\right)}
$$

The instantons pressure change $\left(\partial \mathrm{P}_{(\mathrm{t})}\right)$ experienced by the vessel segment can be expressed as given below, by differentiating (2) with respect to time $(\partial t)$.

$$
\partial \mathrm{P}_{(\mathrm{t})}=\mathrm{P}_{(\mathrm{t})} \beta \frac{\partial \mathrm{D}_{(\mathrm{t})}}{\mathrm{D}_{\mathrm{D}}}
$$

Equation (3) proves the inherent relationship of arterial pressure gradient with geometrical and material properties of vessel segments. The well-established Bramwell-Hill (BH) equation [9], on the other hand, describes the arterial pressure change $\partial \mathrm{P}_{(\mathrm{t})}$ in terms of distension $\partial \mathrm{D}_{(\mathrm{t})}$, blood density $(\rho)$ and incremental local $\operatorname{PWV}\left(\mathrm{C}_{(\mathrm{t})}\right)$ as:

$$
\partial \mathrm{P}_{(\mathrm{t})}=2 \rho \mathrm{C}_{(\mathrm{t})}^{2} \frac{\partial \mathrm{D}_{(\mathrm{t})}}{\mathrm{D}_{(\mathrm{t})}}
$$

Substituting (4) into (3) gives the formulation in (5), which is referred to as the 'P $\beta$-model' [5].

$$
P_{(t)} \beta=\frac{2 \rho C_{(t)}^{2} D_{D}}{D_{(t)}}
$$

By evaluating the $\mathrm{P} \beta$-model for the diastolic pressure condition of a given cardiac cycle and dividing it by (5), an expression for $\mathrm{C}_{(\mathrm{t})}$ can be derived as in (6); in which, $\mathrm{C}_{\mathrm{D}}$ is the local $\mathrm{PWV}$ corresponding to $\mathrm{P}_{\mathrm{D}}$.

$$
C_{(t)}^{2}=C_{D}^{2} \frac{P_{(t)}}{P_{D}} \frac{D_{(t)}}{D_{D}}
$$

Further, by replacing pressure terms from (6) using (2), we have developed a new mathematical model, as given in (7), for the assessment of incremental local PWV, in terms of $C_{D}, \beta$, and arterial diameter parameters.

$$
C_{(t)}=C_{D}\left(\sqrt{\frac{D_{(t)}}{D_{D}}}\right) e^{\beta / 2\left(\frac{D_{(t)}}{D_{D}}-1\right)}
$$

This formulation describes the non-linear relationship and the dependency of instantaneous local PWV with the arterial dimensions and material properties. Therefore, plotting the estimated $\mathrm{C}_{(\mathrm{t})}$ against $\mathrm{D}_{(\mathrm{t})}$ measured from the target artery gives the locus and non-linear characteristics of the incremental local PWV from each cardiac cycle.

\subsection{Method for practical implementation}

Although non-invasive assessment of arterial diameter waveform and intermediate pressure values are practically feasible, measurement of $\beta$ and $C_{D}$ is not straightforward. While continuous diameter waveform can be obtained from any artery using medical imaging modalities such as ultrasound, the snapshot of systolic and diastolic pressure from the brachial artery can be directly measured using a sphygmomanometer and upper arm pressure-cuff. Note that, the scaling of brachial pressure values to any target arterial site using its continuous diameter waveform is well-established with acceptable accuracy [6]. Therefore, as described below, $\beta$ and $C_{D}$ from each cardiac cycle can be evaluated in real-time by directly measuring; (i) luminal diameter waveform $\mathrm{D}_{(\mathrm{t})}$, (ii) systolic pressure $\mathrm{P}_{\mathrm{S}}$, and (iii) diastolic pressure $\mathrm{P}_{\mathrm{D}}$ from the target arterial sites.

Knowing the end-diastolic and systolic peak diameter ( $D_{D}$ and $D_{S}$ respectively) from $D_{(t)}$ for each cardiac cycle, the stiffness index $\beta$ can be evaluated in a beat-by-beat manner using corresponding $P_{S}$ and $P_{D}$ values as [5]:

$$
\beta=\frac{\ln \left(\frac{P_{\mathrm{S}}}{\mathrm{P}_{\mathrm{D}}}\right)}{\left(\frac{\mathrm{D}_{\mathrm{S}}}{\mathrm{D}_{\mathrm{D}}}-1\right)} .
$$

Now, the absolute value of $\mathrm{C}_{\mathrm{D}}$ (local PWV at diastolic pressure level) corresponding to each cardiac cycle can be evaluated with the help of the proposed $\mathrm{P} \beta$-model as [5]:

$$
C_{D}=\sqrt{\frac{P_{D} \beta}{2 \rho}} .
$$

Hence, by using $\beta$ and $C_{D}$ for individual cardiac cycles, one can perform a beat-by-beat evaluation of the locus of incremental local PWV by employing the newly proposed empirical model (7), using the non-invasively measurable physiological parameters $\left(\mathrm{D}_{(\mathrm{t})}, \mathrm{P}_{\mathrm{S}}\right.$, and $\left.\mathrm{P}_{\mathrm{D}}\right)$.

\section{Material and methods}

\subsection{Assessment of reference measurement}

In order to evaluate the accuracy and reliability of the proposed empirical model, reference measurements were obtained by implementing the $\mathrm{BH}$ equation given in (4). This approach is recognized as the reference-standard for local PWV measurement [10]. However, unlike the newly proposed empirical model (7), direct implementation of the $\mathrm{BH}$ equation for incremental local PWV evaluation is challenging. The primary reason is that the $\mathrm{BH}$ equation holds only for hyperelastic vessels, in the absence of viscous properties of the vessel wall and pressure wave reflections [9]. Presence of viscous properties results in hysteresis in the pressure-diameter variation. Hence, we have implemented a viscoelastic model of the artery to quantify the viscosity index $(\eta)$ and extract the elastic component of the pressure $\left(\mathrm{P}_{\mathrm{E}(\mathrm{t})}\right)$, as in (10).

$$
\mathrm{P}_{\mathrm{E}(\mathrm{t})}=\mathrm{P}_{(\mathrm{t})}-\eta \frac{\partial \mathrm{D}_{(\mathrm{t})}}{\partial \mathrm{t}}
$$

The value of $\eta$ for individual cardiac cycles can be estimated by iterative optimization to minimize the pressure-diameter hysteresis. Finally, reference $\mathrm{C}_{(\mathrm{t})}$ can be evaluated using $\mathrm{P}_{\mathrm{E}(\mathrm{t})}$ in the $\mathrm{BH}$ equation as in (11).

$$
\mathrm{C}_{(\mathrm{t})}=\sqrt{\frac{\mathrm{D}_{(\mathrm{t})}}{2 \rho} \frac{\partial \mathrm{P}_{\mathrm{E}(\mathrm{t})}}{\partial \mathrm{D}_{(\mathrm{t})}}}
$$




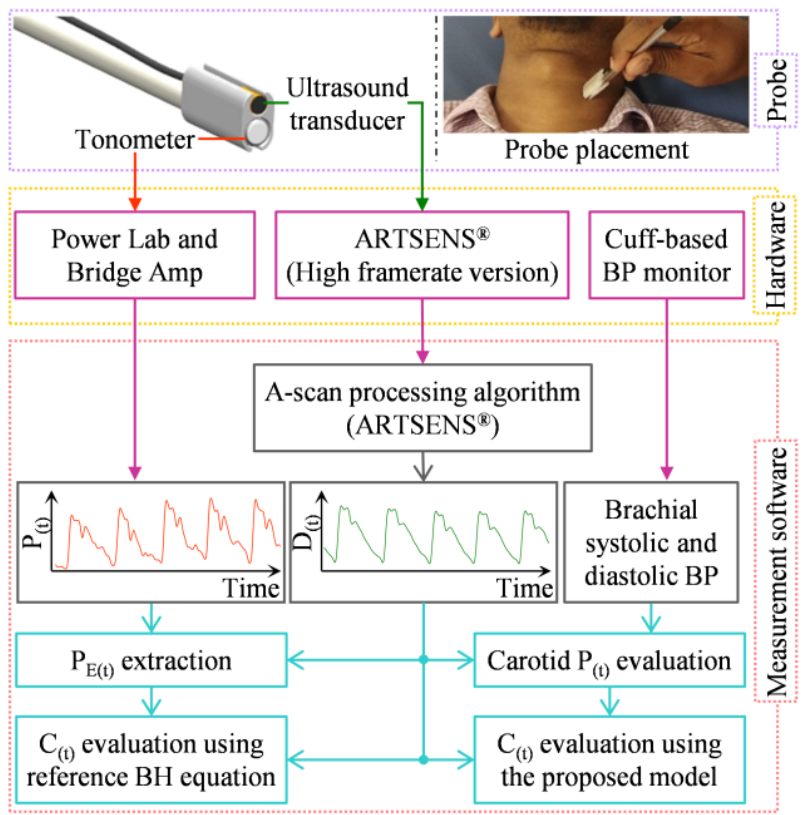

Figure 1. Architecture of the developed measurement system. The inset shows probe placement at the carotid.

\subsection{In-vivo instrumentational setup}

Fig. 1 illustrates the instrumentational setup developed for in-vivo validation of the developed incremental local PWV model. The measurement system employed a handheld probe with a focused, broadband single-element ultrasound transducer (center-frequency $=5 \mathrm{MHz}$ ). Our extensively validated image-free ultrasound technology, ARTSENS $^{\circledR}$ [11], was used for measuring $D_{(t)}$ from the carotid artery by processing the captured ultrasound Ascan frames (framerate $=1 \mathrm{kHz}$ ). Systolic and diastolic brachial BP measured from the upper arm using a clinical-grade sphygmomanometer $\quad\left(\operatorname{SunTech}^{\circledR} \quad 247^{\mathrm{TM}}\right.$ ) was scaled to the carotid artery [6] to obtain the carotid $P_{S}$ and $P_{D}$ in real-time. For each cardiac cycle, by evaluating $C_{D}$ and $\beta$, the incremental local PWV was obtained using the proposed mathematical model with the help of a fully automated custom measurement software.

As depicted in Fig. 1, a calibrated tonometer (Millar SPT 301) was also integrated into the developed handheld probe. This unique arrangement was made for the current study to capture $\mathrm{P}_{(t)}$ along with $\mathrm{D}_{(\mathrm{t})}$ from the same arterial site, to measure reference incremental local PWV using $\mathrm{BH}$ equation-based method. Optimal arrangement of tonometer-ultrasound transducers (separation distance $=2 \mathrm{~mm}$ ) ensured single-site measurement of pressure and diameter waveforms. Simultaneous acquisition of $\mathrm{P}_{(\mathrm{t})}$ and $\mathrm{D}_{(\mathrm{t})}$ was performed by synchronizing the analog front-end of tonometer (ADI's Power Lab and Bridge Amp) with the ARTSENS ${ }^{\circledR}$ device. Post-elimination of the viscous pressure from $\mathrm{P}_{(\mathrm{t})}$, the reference incremental local PWV was evaluated for each cardiac cycle.

\subsection{Data collection}

An in-vivo study was performed on 15 healthy subjects (mean age $=32 \pm 5$ years). The study objective was to evaluate the carotid incremental local PWV and its locus using the proposed mathematical model and compare the obtained traces against the reference method, and to examine the accuracy and reliability of the newly proposed method. The study was carried out in compliance with the Helsinki Declaration. Written informed consent was obtained from the recruited subjects after explaining the study objectives.

All the physiologic measurements were obtained from the recruited subjects in the supine posture, post $5-10$ minutes of rest. The resting BP from the brachial artery was initially recorded and fed into the measurement software. The ultrasound-tonometer probe was placed on the left side of the neck above the carotid artery after identifying the carotid pulse site by palpation. Desired signals were continuously recorded ( $\sim 25$ cardiac cycles), and beat-by-beat incremental local PWVs were obtained using both the proposed and reference methods.

\section{Experimental results and discussion}

High-fidelity non-invasive carotid $\mathrm{D}_{(\mathrm{t})}$ waveforms (and $\mathrm{P}_{(\mathrm{t})}$ for the reference method) were continuously captured from the subjects, and yielded reliable measurements of incremental local PWV, $\mathrm{C}_{(\mathrm{t})}$, over continuous cardiac cycles. Having determined $\mathrm{C}_{(\mathrm{t})}$ values for the anacrotic phase of each cardiac for all the subjects, we traced their loci using the proposed mathematical model and compared with the reference method. Fig. 2 illustrates representative examples of the measured $\mathrm{C}_{(t)}$ plotted against corresponding $\mathrm{D}_{(\mathrm{t})}$, for a single cardiac cycle per subject. Similar results were obtained for all the recruited subjects. The locus of $\mathrm{C}_{(\mathrm{t})}$ measured using the proposed model closely followed the $\mathrm{BH}$ equation-derived instantaneous local PWV values. Root-mean-square-error lesser than $0.05 \mathrm{~m} / \mathrm{s}$ for $\mathrm{C}_{(\mathrm{t})}$ and 0.07 for $\beta$ were observed between the measured and reference values. These results establish the accuracy and reliability of the proposed model and its practical implementation method.

When considering the systolic phase of the cardiac cycle, the local PWV values increase significantly (refer Fig. 2) as the pressure changes from $P_{D}$ to $P_{S}$. The application of developed model allows measurement of absolute change $\left(\Delta \mathrm{C}_{(\mathrm{t})}\right)$ and the degree of nonlinearity (an indicator arterial stiffness [6]) in local PWV within a cardiac cycle using $\mathrm{D}_{(\mathrm{t})}$ and brachial $\mathrm{BP}$ alone. The measured $\Delta \mathrm{C}_{(\mathrm{t})}$ and $\beta$ were in the range of $0.46-1.27 \mathrm{~m} / \mathrm{s}$ and $1.98-4.33$ respectively. Typically, elevated $\Delta \mathrm{C}_{(\mathrm{t})}$ values were observed for subjects with relatively stiffer arteries (carotid $\beta$ was relatively high in such cases). These results comply with a recent study conducted on a 


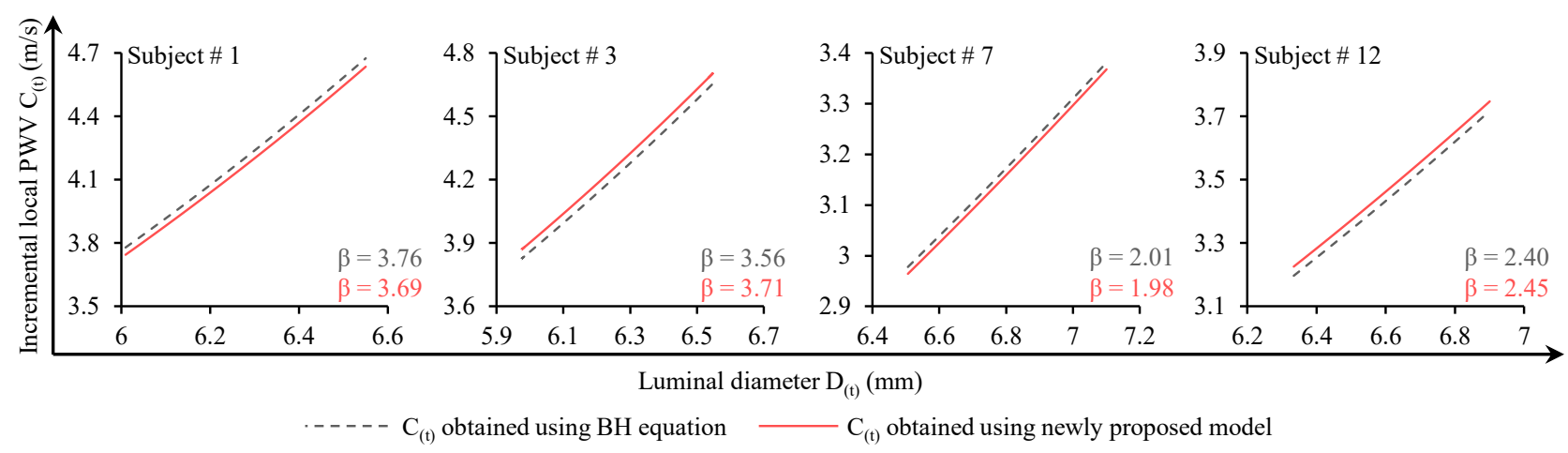

Figure 2. Examples of the locus of carotid incremental local PWV measured over a single cardiac cycle using the newly proposed mathematical model and the reference Bramwell-Hill equation-based method.

large cohort of 1776 individuals [3]. Their study provided clinical evidence of the association of $\Delta \mathrm{C}_{(\mathrm{t})}$ with left ventricular mass index. Note that, due to the absence of a suitable methodology, $\Delta \mathrm{C}_{(\mathrm{t})}$ was obtained by subtracting the systolic and diastolic local PWVs [3]. However, in [3], the investigators strongly recommend using $\Delta \mathrm{C}_{(\mathrm{t})}$ in conjunction with instantaneous values of incremental local PWV for comprehensive cardiovascular risk prediction. A study on 102 healthy individuals and 37 patients affected by the vascular Ehlers-Danlos syndrome demonstrated the importance of tracing the locus of $\mathrm{C}_{(\mathrm{t})}$ to go beyond the conventional single-value $P W V$ to better characterize arterial pressure and stiffness changes concerning age or other collagen alterations [4]. On a similar note, we have recently developed a calibrationfree cuffless central arterial BP monitor by exploring the concept of incremental local PWV [5].

Future efforts are indeed required to perform extensive validation of the proposed novel approach over a wide range of pathophysiological conditions, and to bring the developed system to practice.

\section{Conclusion}

In this work, a new mathematical model which utilize arterial diameter waveform for the determination of the incremental local PWV and its locus was presented. The theoretical analysis showed the dependency of local PWV on arterial dimensions and stiffness level. It was validated in-vivo using a custom measurement system, and demonstrated its accuracy and practical feasibility. The proposed approach based on monitoring of instantaneous variations in local PWV has the potential to revolutionize the current cardiovascular risk stratification techniques.

\section{References}

[1] T. Pereira et al., "Novel methods for pulse wave velocity measurement," J. Med. Biol. Eng., vol. 35, no. 5, pp. 555$565,2015$.

[2] P. M. Nabeel et al., "Local pulse wave velocity: theory, methods, advancements, and clinical applications," IEEE Rev. Biomed. Eng., pp. 1-39, 2019.

[3] E. Hermeling et al., "The change in arterial stiffness over the cardiac cycle rather than diastolic stiffness is independently associated with left ventricular mass index in healthy middle-aged individuals," J. Hypertens., vol. 30, no. 2, pp. 396-402, 2012.

[4] T. Mirault et al., "Carotid stiffness change over the cardiac cycle by ultrafast ultrasound imaging in healthy volunteers and vascular Ehlers-Danlos syndrome," J. Hypertens., vol. 33, no. 9, pp. 1890-1896, 2015.

[5] P. M. Nabeel et al., "Bi-modal arterial compliance probe for calibration-free cuffless blood pressure estimation," IEEE Trans. Biomed. Eng., vol. 65, no. 11, pp. 2392-2404, 2018.

[6] J. M. Meinders and A. G. Hoeks, "Simultaneous assessment of diameter and pressure waveforms in the carotid artery," Ultrasound Med. Biol., vol. 30, no. 2, pp. 147-154, 2004.

[7] K. Hayashi et al., "Stiffness and elastic behavior of human intracranial and extracranial arteries," J. Biomech., vol. 13, no. 2 , pp. $175-179,181-184,1980$.

[8] K. Hayashi et al., "Clinical assessment of arterial stiffness with cardio-ankle vascular index: theory and applications," J. Hypertens., vol. 33, no. 9, pp. 1742-1757, 2015.

[9] J. C. Bramwell and A. V Hill, "The velocity of the pulse wave in man," R. Soc. Publ., vol. 93, no. 652, pp. 298-306, 1922.

[10] P. Segers et al., "Wave reflection leads to over- and underestimation of local wave speed by the PU- and QAloop methods: theoretical basis and solution to the problem," Physiol. Meas., vol. 35, no. 5, pp. 847-861, 2014.

[11] J. Joseph et al., "Technical validation of ARTSENS-an image free device for evaluation of vascular stiffness," IEEE J. Transl. Eng. Heal. Med., vol. 3, pp. 1-13, 2015.

Address for correspondence:

Dr. Nabeel P M

Research Scientist (Cardiovascular Science)

Healthcare Technology Innovation Centre (HTIC), IIT Madras

5C, 5th Floor, Phase II - IIT Madras Research Park

Kanagam Road, Taramani, Chennai, India - 600113

Email: nabeelnpm@gmail.com,nabeel@htic.iitm.ac.in 